\title{
BMJ Open Association among emotional and behavioural problems, sexual minority status and suicidal behaviours in adolescents: a cross-sectional study from Guangdong Province in China
}

\author{
Wenyan Li, ${ }^{1}$ Lan Guo, ${ }^{1}$ Sheng Zhang, ${ }^{1}$ Wanxin Wang, ${ }^{1}$ Xiaoliang Chen, ${ }^{1}$ Qian Li, ${ }^{1}$ \\ Ciyong Lu (D) ${ }^{1,2}$
}

To cite: Li W, Guo L, Zhang S, et al. Association among emotional and behavioura problems, sexual minority status and suicidal behaviours in adolescents: a crosssectional study from Guangdong Province in China. BMJ Open 2021;11:e051485. doi:10.1136/ bmjopen-2021-051485

- Prepublication history and additional supplemental material for this paper are available online. To view these files, please visit the journal online (http://dx.doi.org/10.1136/ bmjopen-2021-051485).

Received 22 March 2021 Accepted 31 August 2021

A) Check for updates

(C) Author(s) (or their employer(s)) 2021. Re-use permitted under CC BY-NC. No commercial re-use. See rights and permissions. Published by BMJ.

${ }^{1}$ Department of Medical Statistics and Epidemiology, School of Public Health, Sun Yat-Sen University, Guangzhou, Guangdong, China

${ }^{2}$ Guangdong Provincial Key Laboratory of Food, Nutrition and Health, Sun Yat-Sen University, Guangzhou, Guangdong, China

Correspondence to

Dr Ciyong Lu;

luciyong@mail.sysu.edu.cn

\section{ABSTRACT}

Objectives This study was conducted to estimate whether emotional and behavioural problems mediate the association between sexual minority status and suicidal behaviour among Chinese adolescents. We also tested whether such mediation is moderated by the sex assigned at birth.

Design Cross-sectional observational study.

Setting A secondary analysis of the cross-sectional data collected from 7th-grade to 12th-grade students in junior high schools $(n=36)$, senior high schools $(n=24)$ and vocational high schools $(n=12)$ in six cities of Guangdong Province, China. A multistage, stratified cluster, random sampling method was used in the 2019 School-based Chinese Adolescents Health Survey.

Participants A total of 16663 students aged 11-20 years.

Outcome measures Two main psychological and mental health outcomes: (1) self-reported version of the Strengths and Difficulties Questionnaire to measure emotional and behavioural problems; and (2) presence of suicidal ideation and suicide attempts in the past 12 months.

Results Emotional and behavioural problems (peer problems, emotional problems, conduct problems and hyperactivity) partially mediated the effects of sexual minority status on suicidal ideation (indirect effect $=0.020$, $95 \% \mathrm{Cl} 0.015$ to 0.025 ) and suicide attempts (indirect effect $=0.012,95 \% \mathrm{Cl} 0.009$ to 0.016 ), accounting for $18.18 \%$ and $14.46 \%$ of the total effect, respectively. Further moderated mediation analyses revealed a greater risk of suicidality among sexual minority girls.

Conclusions Emotional and behavioural problems partially explained the increased risk of suicidality among sexual minority adolescents, and appreciably higher among sexual minority girls than boys. To prevent subsequent suicidality, the risks of emotional and behavioural problems in sexual minority adolescents should be identified at an early stage and reduced by means of preventive measures.

\section{INTRODUCTION}

Suicide is a severe public health problem that leads to more than 700000 deaths every year

\section{STRENGTHS AND LIMITATIONS OF THIS STUDY}

$\Rightarrow$ This is a large-scale, representative study of Chinese adolescents' sexual attraction and mental health.

$\Rightarrow$ Structural equation modelling was used to explore the mediating role of emotional and behavioural problems.

$\Rightarrow$ This is a detailed analysis of sex differences in the association among sexual attraction, suicidal behaviours, and emotional and behavioural problems.

$\Rightarrow$ Gender dysphoria/transgender status was not assessed in this study.

$\Rightarrow$ Due to the cross-sectional design, causal inferences cannot be inferred.

in the world. ${ }^{1}$ Adolescents are in a critical developmental vulnerability period, placing them at higher risk for suicidal behaviours. ${ }^{2}$ In China, suicide is the fourth leading cause of death among adolescents aged 10-19, with a suicide rate of 2.53 per 100000 individuals in $2016 .^{3}$ In recent decades, there has been unprecedented concern regarding the mental health of sexual minority adolescents (SMAs; ie, adolescents who experience same-sex attraction, engage in same-sex sexual behaviour, or self-identify as gay, lesbian or bisexual). SMAs are more likely to be exposed to violence, isolation, discrimination and stigmatisation, associated with an increased risk of suicidal behaviours, compared with their heterosexual peers. ${ }^{45}$ On average, $28 \%$ of SMAs have a history of suicidality and they are two to three times more likely to have suicidal ideation and suicide attempts compared with heterosexual adolescents. ${ }^{67}$ Thus, there is an urgent need to prevent the occurrence of suicidal behaviours in SMAs through more specific studies that assess their characteristics and elucidate the risk factors of suicidality. 
A large number of studies have shown that SMAs suffer from a variety of mental and behavioural health issues. ${ }^{8-11}$ According to the Meyer minority stress model, ${ }^{4}$ sexual minority individuals face higher levels of social stressors that are both objective (prejudice and discrimination) and subjective (internalised homophobia), which result in more negative health outcomes, such as mental disorders (eg, mood and anxiety disorders) and emotional and behavioural problems (ie, internalising and externalising problems). Previous studies reported that SMAs suffered from more severe emotional and behavioural problems, including conduct, hyperactivity, depression and anxiety disorders. ${ }^{89}$ Research also demonstrated that emotional and behavioural problems were associated with suicidal behaviours among adolescents. ${ }^{10}$ Moreover, recent reports suggested that Chinese SMAs were at an increased risk of behavioural problems, such as smoking, frequent drinking ${ }^{12}$ and drug abuse,${ }^{13}$ compared with heterosexual adolescents. Multiple studies have found that Chinese SMAs were anywhere from two to four times more likely to engage in emotional problems and suicidal behaviours, including anxiety and depressive symptoms, suicidal ideation, and suicide attempts, than heterosexuals. ${ }^{11} 14$ However, a limited number of studies have examined the mediating role of emotional and behavioural problems with respect to sexual minority status and suicidality. In addition, due to the difficulties of recruiting adequate sexual minority volunteers, the sample sizes of prior studies were relatively small.

Furthermore, sex differences likely play a major role in mental health disparities among SMAs. Sex is the sex assigned at birth (boy or girl) by a physician, generally based on chromosomes, gonads and anatomy. ${ }^{15}$ In this article, sex is used synonymously with the sex assigned at birth. The minority stress model proposes that minority stressors related to one's sexual identity are unique to sexual minority individuals. ${ }^{4}$ Biobehavioural responses differ by sex when coping with stress, which shapes the risk of mental disorders. ${ }^{16}$ A recent systematic review revealed that some studies reported elevated risks of depression, anxiety and suicidal behaviours in sexual minority men than in women, but some others conclude the opposite. ${ }^{17}$ Research in Chinese SMAs showed that girls had greater tendency for emotional problems and suicidal ideation, while boys had greater tendency for suicide attempts. ${ }^{11}$ However, it remains unclear whether sex assigned at birth plays a moderating role in the impact of emotional and behavioural problems on suicidality in sexual minorities.

Additionally, the Chinese cultural background is deeply influenced by Confucianism, in which people are expected to marry and have children. ${ }^{18}$ It has been reported that external pressure to get married was significantly associated with poorer mental health via psychological functions among sexual minority individuals, which can be explained by the theory of psychological mediation framework. ${ }^{19}$ Therefore, there might be differences in the associations of emotional and behavioural problems with suicidal behaviours among SMAs in China and other Western countries. Thus, we conducted this large-scale study to examine the role of emotional and behavioural problems in the associations of sexual minority status and suicidal behaviours and whether these associations vary between sexes. Here, we outlined two primary hypotheses. The first hypothesis was that the associations between sexual minority status, suicidal ideation and suicide attempts were mediated by emotional and behavioural problems. The other was that these associations might be moderated by sex assigned at birth.

\section{METHODS}

\section{Study design and participants}

Data were drawn from the 2019 School-based Chinese Adolescents Health Survey (SCAHS), ${ }^{14}{ }^{20}$ an ongoing large-scale, cross-sectional study about health-related behaviours among Chinese adolescents ( 7 th-12th grade), which has been conducted every 2 years since 2007. A multistage, stratified cluster, random sampling method was used in the 2019 SCAHS. The procedures for data collection were as follows. In stage 1, Guangdong Province was divided into three economic strata (high level, middle level and low level) by gross domestic product per capita, and then two cities were randomly selected as representatives from each stratum. In stage 2 , six junior high schools (ie, grades 7-9), four senior high schools (ie, grades 10-12) and two vocational high schools (ie, grades 10-12, schools providing specific vocational training) were randomly selected from each representative city. In stage 3 , two classes were randomly chosen from each grade within the selected schools, and all available students were invited to voluntarily participate in the study from the selected classes. All data were collected from November 2018 to January 2019. In total, 20370 students completed our questionnaires (a response rate of $96.9 \%$ ). Because we were primarily interested in the suicidality of students who acknowledged and reported their sex attraction, students belonging to the 'unsure' category $(n=826)$ and 'refuse to answer' category $(\mathrm{n}=2881$ ) were not included in the present analysis. Therefore, a total of 16663 students were ultimately included in the present analysis.

\section{Procedure}

A standardised self-reported questionnaire was completed in the classroom during a normal class period (40 or $45 \mathrm{~min}$ ). To increase the response rate of stigmatised behaviours and minimise potential information bias, the questionnaires were anonymous and our research assistants organised the investigation instead of the teachers. Written informed consent was obtained from each student who was at least 18 years old and volunteered to participate in the study. If the student was under 18 years of age, written informed consent was obtained from the student's parents (or legal guardians).

\section{Patient and public involvement}

Patients or the public were not involved in the development of the research question, study design or data interpretation in this study. 


\section{Measures}

Sexual minority status

Sexual minority status was measured by asking the following question regarding sexual attraction: 'When in a romantic relationship to whom you are attracted?' The response options included 'a boy', 'a girl', 'both boy and girl', 'neither boy nor girl', 'unsure' and 'refuse to answer'. When a girl chose the option 'a girl' or 'both boy and girl' and a boy chose the option 'a boy' or 'both boy and girl', they were classified as sexual minorities. These questions were taken from Russell and Joyner, ${ }^{21}$ and they have been confirmed to be more suitable for measuring same-sex orientation in adolescents than other methods and have been used in many relevant studies. ${ }^{22}$

\section{Suicidal ideation and suicide attempts}

The following two questions were used to assess participants' suicidal ideation and suicide attempts, respectively: 'During the past 12 months, how many times did you think seriously about committing suicide?' and 'During the past 12 months, how many times did you actually attempt suicide deliberately? ${ }^{23}$ Available responses were zero, once and at least two times, and the latter two were combined into an 'attempted' group in the moderated mediation models.

\section{Emotional and behavioural problems}

Emotional and behavioural problems were measured by the self-reported version of the Strengths and Difficulties Questionnaire (SDQ), ${ }^{24}{ }^{25}$ the most widely used multidimensional mental health screening instrument for children and adolescents, which has good reliability and validity when used among Chinese adolescents. ${ }^{26} 27$ The SDQ is composed of 25 items, divided into 5 scales: emotional problems, conduct problems, hyperactivity, peer problems and prosocial behaviour. In this study, 'total difficulties' is measured by the first four subscales, and the prosocial behaviour subscale scores separately from SDQ. Response options were given on a 3-point Likert scale ( $1=$ not true, $2=$ somewhat true, $3=$ certainly true). Higher scores in each subscale predict a greater level of emotional and behavioural problems, but the prosocial behaviour subscale is scored inversely by comparison. The Cronbach's alpha for the total difficulties score was 0.76 and for the prosocial behaviour was 0.75 in the present study, which indicated sufficient internal consistency.

\section{Covariates}

The covariates in the present study included factors that have been previously reported to be associated with suicidal behaviours among SMAs. ${ }^{21} 28{ }^{29}$ First, the Center for Epidemiological Studies Scale (CES-D) was used to measure symptoms of depression. ${ }^{30}$ The scale has been validated in Chinese and has been extensively used among Chinese populations of different ages. ${ }^{31} 32$ The CES-D is a 20-item self-reported questionnaire, with each item rated on a scale from 'rarely or none of the time $=1$ ' to 'most or all of the time $=4$ '. Higher scores indicate more severe depressive symptomology, with total scores ranging from 0 to 60. A standard cut-off point of 16 or more for CES-D is used to identify participants with relevant clinical depressive symptoms. ${ }^{33}$ In this study, the Cronbach's alpha was 0.89 .

Second, demographic characteristics including sex assigned at birth (boy or girl), age, living arrangements (with both parents or not), family relationships (good relationships or not) and household socioeconomic status (HSS) were measured. HSS was measured by asking students about their perception of their family's economic status (categorised into 'excellent or very good' $=1$, 'good'=2 and 'fair or poor' $=3$ ). Those who selfreported smoking cigarettes (or drinking alcohol) on 1 or more days during the last month were considered current smokers (or current drinkers). ${ }^{34}$

\section{Statistical analysis}

First, differences between sexual minority and heterosexual adolescents were examined using independent sample t-tests or, where data were in the form of proportions, using Rao-Scott $\chi^{2}$ tests. Second, to investigate the variables contributing to suicidal behaviours, univariate and multivariate logistic regression models were generated. Third, the mediation and moderated mediation models were analysed using the structural equation modelling with a maximum likelihood estimator. ${ }^{35}$ Path analysis was used for simultaneous testing of both direct and mediating effects, and the hypothesised model was tested in two ways. ${ }^{36}$ First, we assessed the fit of a mediation model without the hypothesised interaction to validate whether the association between sexual minority status and suicidal behaviours was mediated by emotional and behavioural problems (figure 1A). Next, we tested the moderated mediational model with latent interaction, that is, whether sex assigned at birth moderated the effects of sexual minority status on suicidal behaviours (figure 1B).

Several commonly acknowledged model fit indices were recommended: comparative fit index (CFI), standardised root mean square residual (SRMR) and root mean square error of approximation (RMSEA), which indicated a satisfactory fit (if CFI $>0.90$, SRMR $<0.08$, RMSEA $<0.05$ ). ${ }^{37}$ The bias-corrected $95 \%$ CI was estimated with 5000 bootstrapping resamples. ${ }^{38}$ Statistical significance was defined as a twotailed $\mathrm{p}$ value $<0.05$. Missing data in the analysis accounted for $<3.0 \%$ for all relevant variables, so observations with missing data were eliminated. All statistical analyses were conducted using IBM SPSS V.25.0 and Mplus V.8.0 (Muthén and Muthén, Los Angeles, California, USA).

\section{RESULTS}

Demographic characteristics stratified by sexual minority status

The characteristics of the sample are shown in online supplemental table 1 . Of the 16663 students analysed, $90.1 \%$ and $9.9 \%$ self-reported as heterosexuals and sexual 

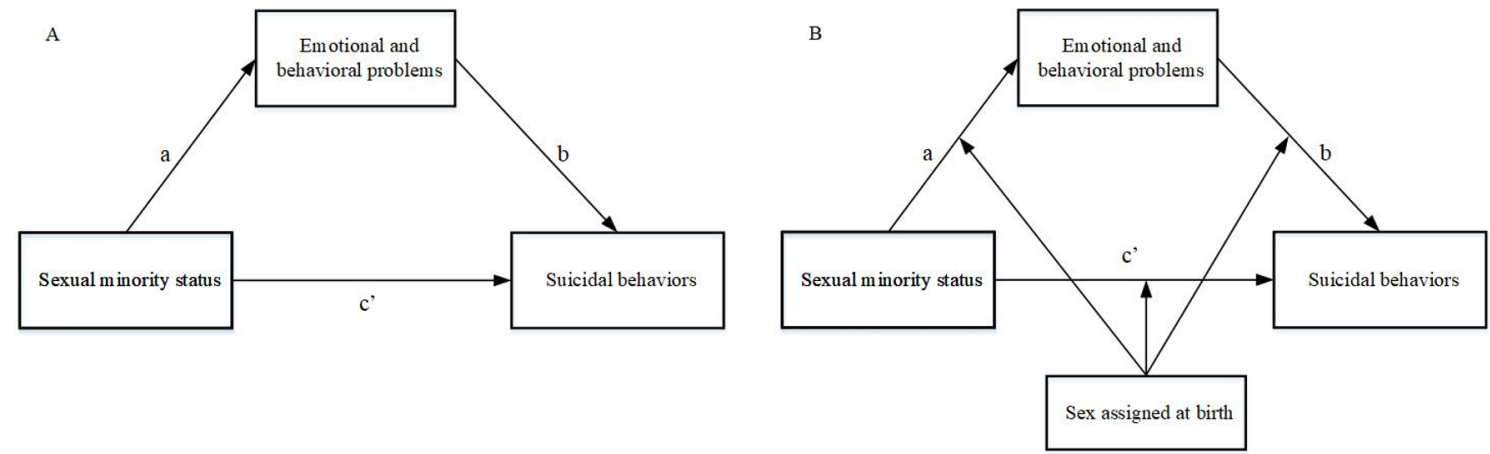

Figure 1 Schematic model of the association between sexual minority status, emotional and behavioural problems, and sex assigned at birth and suicidal behaviours. (A) Emotional and behavioural problems as the mediator. (B) Sex assigned at birth as a moderator of the mediation model. Path $a$, association between independent variable and mediator; path $b$, association between mediator and dependent variables; path c', direct effect of independent variable on dependent variable.

minorities, respectively. Students had a mean age of 15.1 ( $\mathrm{SD}=1.8)$, ranging from 11 to 20 , with 8475 boys $(50.9 \%)$ and 8188 girls $(49.1 \%)$. The mean (SD) SDQ scores for emotional problems, conduct problems, hyperactivity, peer problems, prosocial behaviour and the total difficulties were 2.3 (2.2), 1.9 (1.4), 3.4 (2.1), 2.9 (1.5), 7.4 (2.1) and 10.6 (5.2), respectively. The mean CES-D score of the students was $14.4(\mathrm{SD}=10.2)$. Sexual minority students reported a higher prevalence of depressive symptoms (a cut point of CES-D $\geq 16$ ) and past-year suicidal ideation and suicide attempts $(p<0.001)$. Additionally, the two groups also had significant differences in the distribution of sex assigned at birth, age, family structure, family relationship, alcohol consumption and each subscale of the SDQ $(\mathrm{p}<0.05)$.

\section{Association of sexual minority status, emotional and behavioural problems, and sex assigned at birth with suicidal behaviours}

Without controlling for other variables, sexual minority status, sex assigned at birth, and emotional and behavioural problems were significantly associated with suicidal ideation and suicide attempts (model 1 in table 1).

Multivariable logistic regression models were conducted to adjust for all the covariates, including age, family structure, family relationship, HSS, smoking, drinking and depressive symptoms. The results demonstrated that sexual minority status was positively associated with suicidal ideation (adjusted OR $(\mathrm{aOR})=2.37$, 95\% CI 2.09 to 2.68) and suicide attempts $(\mathrm{aOR}=2.40$, 95\% CI 1.97 to 2.92). The adjusted models also showed that, compared with boys, girls exhibited a higher risk of suicidal ideation ( $\mathrm{aOR}=2.14,95 \%$ CI 1.95 to 2.35$)$ and suicide attempts ( $\mathrm{aOR}=2.29,95 \%$ CI 1.90 to 2.75$)$. Moreover, emotional problems, conduct problems, hyperactivity and total difficulties were associated with an elevated risk of both suicidal ideation and suicide attempts, while peer problems were associated with suicide attempts only, and prosocial behaviour was not associated with suicidal ideation and suicide attempts (see aORs for model 2 in table 1).
Furthermore, to examine whether these associations still existed when the unadjusted multivariate logistic regression models simultaneously contained all five subscales of SDQ, sensitivity analyses were performed. The results showed that the associations of emotional problems, conduct problems and hyperactivity with suicidal ideation and suicide attempts were still statistically significant.

The mediating effects of emotional and behavioural problems Emotional and behavioural problems were divided into total difficulties and prosocial behaviour. Structural equation models were used to examine the potential mediating role of total difficulties and prosocial behaviour in the association of sexual minority status with suicidal ideation and suicide attempts. After controlling for depressive symptoms, smoking, drinking, age and other variables, the results showed that all simple path coefficients were statistically significant, when the total difficulties were considered as a mediator $(\mathrm{p}<0.05$; figure 2$)$. As shown in online supplemental table 2, in model 1, a significant indirect path from sexual minority status to suicidal ideation was observed via total difficulties ( standardised $\beta$ estimate $=0.020,95 \%$ CI 0.015 to 0.025 , $\mathrm{p}<0.001$ ), which accounted for $18.18 \%$ of the total effect. In model 2, the standardised indirect effects on suicide attempts were also statistically significant (standardised $\beta$ estimate $=0.012,95 \%$ CI 0.009 to $0.016, p<0.001$ ), which accounted for $14.46 \%$ of the total effect, suggesting that total difficulties were partial mediators of sexual minority status on suicidal ideation and suicide attempts. In addition, our results showed that when prosocial behaviour was considered as a mediator, none of the indirect effects was statistically significant, with the bootstrapping $95 \%$ CI including 0 (suicidal ideation model: $95 \%$ CI -0.002 to 0.000 ; suicide attempts model: $95 \%$ CI -0.001 to 0.000$)$. Therefore, the model was not mediated by prosocial behaviour. In conclusion, total difficulties partly mediated the association between sexual minority status and suicidal behaviours, but prosocial behaviour did not mediate these associations. 


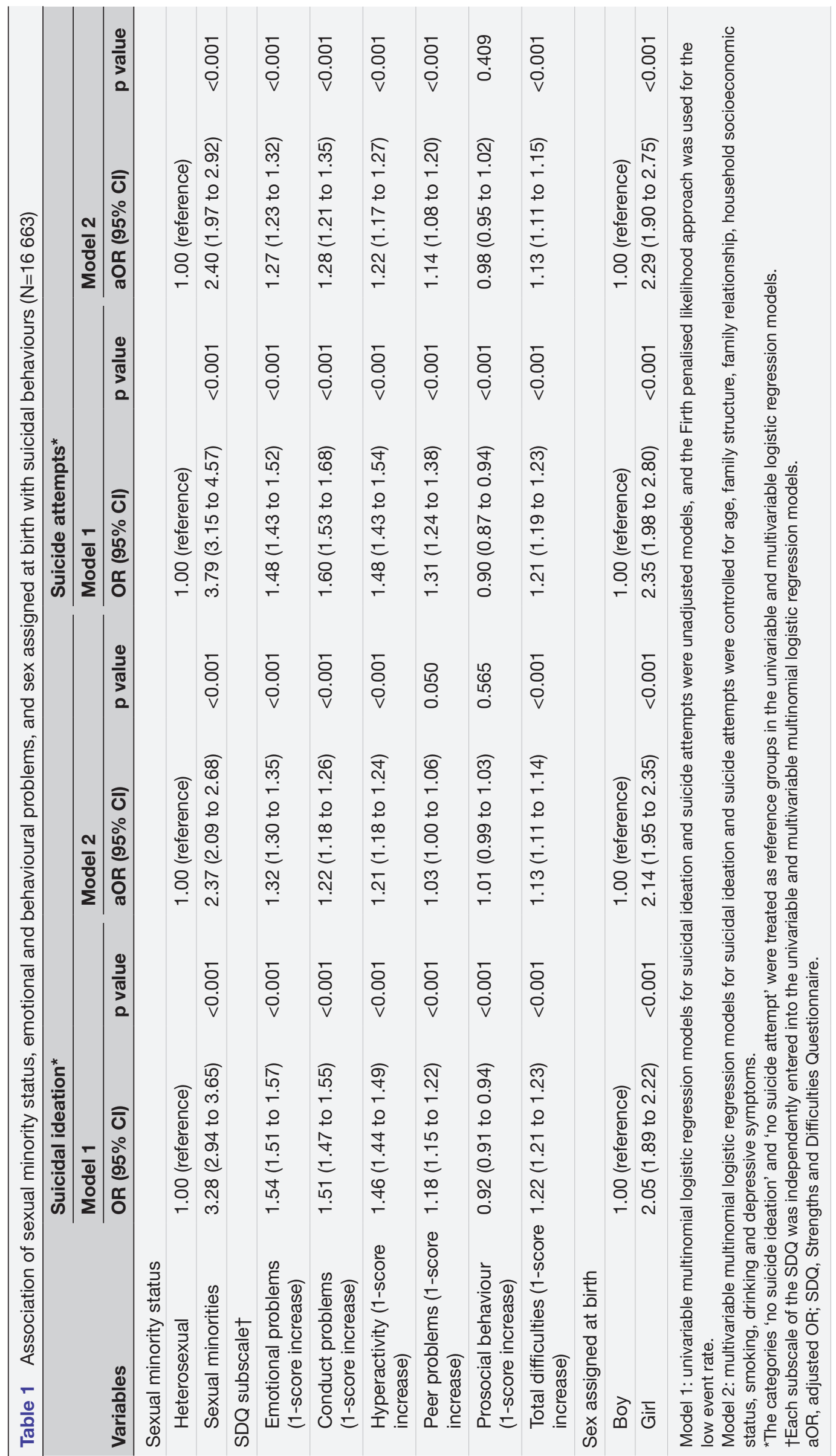




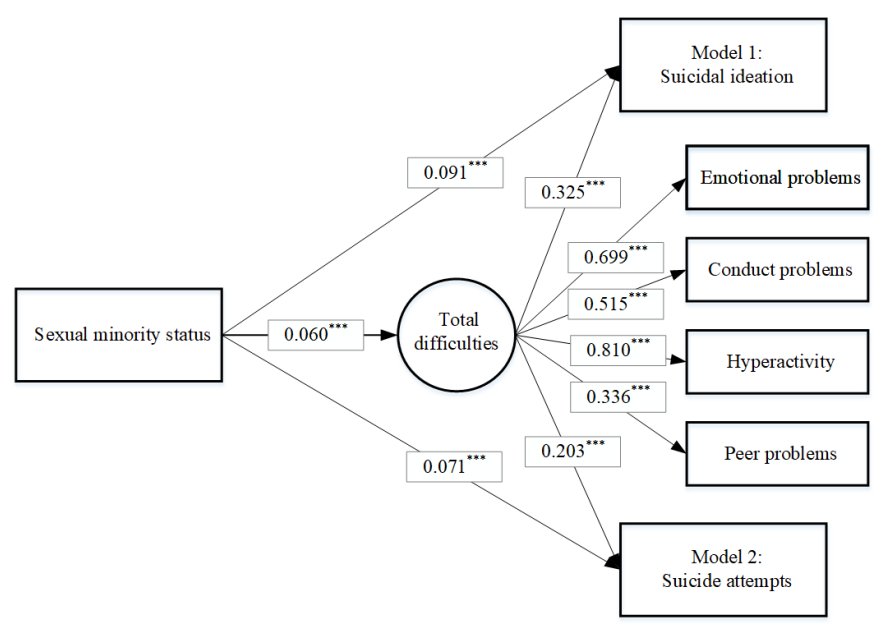

Figure 2 Structural equation model of the relationship between sexual minority status, total difficulties and suicidal behaviours. Model 1 fit indices: $\mathrm{CFI}=0.947$; RMSEA $=0.053$, $95 \% \mathrm{Cl} 0.051$ to 0.056 ; SRMR $=0.028$. Model 2 fit indices: $\mathrm{CFI}=0.946$; RMSEA $=0.050,95 \% \mathrm{Cl} 0.048$ to 0.053 ; SRMR $=0.027$. Unstandardised $\beta$ weights are reported. ${ }^{* \star *} p<0.001$. CFI, comparative fit index; RMSEA, root mean square error of approximation; SRMR, standardised root mean square residual.

\section{The moderated mediation effects of sex assigned at birth}

Moderated mediation analyses were used to examine whether sex assigned at birth exerted moderating influences on the mediating effects of the total difficulties. The results showed that sex assigned at birth moderated the effects of sexual minority status on suicidal ideation $(\beta=0.013, \mathrm{p}<0.001$; model 1 in table 2$)$ and suicide attempts $(\beta=0.005, \mathrm{p}<0.001$; model 2 in table 2$)$ via total difficulties. The interaction pattern is visually depicted in online supplemental figure 1 . The indirect effects between sexual minority status and suicidal ideation via the total difficulties were higher for girls $(\beta=0.029,95 \%$ CI 0.023 to 0.034; model 1 in table 2 and online supplemental figure
1A) than for boys. Similar results were also found using suicide attempts as the dependent variable $(\beta=0.009,95 \%$ CI 0.007 to 0.012 ; model 2 in table 2 and online supplemental figure $1 \mathrm{~B}$ ). The results indicated that sex assigned at birth had a moderating effect both on the direct path (path c') and indirect path (path $\mathrm{a}^{*} \mathrm{~b}$ ) (table 2 and online supplemental figure 2). Online supplemental figure 2 shows the regulatory path of sex assigned at birth in moderated mediation models. Sex assigned at birth moderates the indirect effects through path $\mathrm{b}$ but not path a, so girls have an elevated risk of suicidality, likely because their emotional and behavioural problems exert a more negative influence than they do on boys.

\section{DISCUSSION}

The present study found that after controlling for covariates, SMAs reported greater risks of suicidal ideation and suicide attempts compared with those who identify as heterosexual. This has been confirmed in the broader literature on suicide risk and SMAs. ${ }^{5-7}$ In addition, adolescents with emotional problems, conduct problems and hyperactivity were also at higher risk of suicidal ideation and attempts, which can be appropriately explained by the Meyer minority stress model. ${ }^{4}$ This model indicates that sexual minority individuals are at greater risk of poorer mental health outcomes and suicidality because they face chronic stress. These stressors are likely to also be associated with emotional and behavioural disorders, particularly affective disorders (including depression), hyperactivity and conduct disorders, which are key antecedents to suicidal behaviours. ${ }^{10} 39$ Furthermore, more girls than boys reported a history of suicidal ideation and attempts during the past 12 months. These results are consistent with findings from previous research, suggesting that girls are at increased risk of attempted

Table 2 Conditional direct and indirect effects of sexual minority status on suicidal behaviours through total difficulties in different sexes $(\mathrm{N}=16$ 663)

\begin{tabular}{llll}
\hline Moderator variable & Variables & Model 1: suicidal ideation† & Model 2: suicide attempts† \\
\hline Sex assigned at birth & Effect & & \\
\hline Girl & Direct effect & $0.086(0.072-0.101)^{\star \star \star}$ & $0.032(0.023-0.041)^{\star \star \star}$ \\
\cline { 2 - 4 } & Indirect effect & $0.029(0.023-0.034)^{\star \star \star}$ & $0.009(0.007-0.012)^{\star \star \star}$ \\
\hline Boy & Direct effect & $0.052(0.042-0.063)^{\star \star \star}$ & $0.012(0.007-0.017)^{\star \star \star}$ \\
\cline { 2 - 4 } & Indirect effect & $0.015(0.012-0.019)^{\star \star \star}$ & $0.004(0.003-0.006)^{\star \star \star}$ \\
\hline Difference & Direct effect & $0.034(0.016-0.052)^{\star \star \star}$ & $0.020(0.010-0.030)^{\star \star \star}$ \\
& Indirect effect & $0.013(0.007-0.020)^{\star \star \star}$ & $0.005(0.002-0.008)^{\star}$
\end{tabular}

Model 1 fit indices: $\mathrm{CFI}=0.951$; RMSEA $=0.046,95 \% \mathrm{Cl} 0.044$ to 0.048 ; SRMR=0.029.

Model 2 fit indices: $\mathrm{CFI}=0.947$; RMSEA $=0.044,95 \% \mathrm{Cl} 0.042$ to 0.047 ; SRMR $=0.029$.

$\beta$ weights are reported.

${ }^{*} \mathrm{p}<0.05,{ }^{* * *} \mathrm{p}<0.001$.

†The moderated mediation models for suicidal ideation and suicide attempts were controlled for age, family structure, family relationship, household socioeconomic status, smoking, drinking and depressive symptoms.

$\mathrm{CFI}$, comparative fit index; RMSEA, root mean square error of approximation; SRMR, standardised root mean square residual. 
suicide. ${ }^{40}$ However, it also differs from research that reported a higher 12-month risk of suicidal ideation in sexual minority girls and a similar 12-month risk of suicide attempts for both boys and girls. ${ }^{41}$ Therefore, the interaction between sex and sexual orientation with regard to emotional/behavioural problems and suicidality was also examined in this study.

Our mediation analysis showed that emotional and behavioural problems independently mediated the relationship between sexual minority status and suicidal behaviours, while prosocial behaviour did not. When sex was included as a moderator of these relations, the indirect effect through emotional and behavioural problems was still significant in both boys and girls, and the indirect effect of the latter was greater. Thus, it appears that the mediating effect of emotional and behavioural problems and the moderating effect of sex may be one of the mechanisms of the relationship between sexual minority status and suicidal behaviours to some degree, even though the mechanism of suicidality among SMAs today is still complex and not entirely clear. Moreover, this pattern could be related to differences in the prevalence of emotional and behavioural problems (ie, internalising and externalising problems) between the two sexes. Prior studies indicated that girls reported more total and internalising problems than boys after exposure to adversity, whereas boys were more likely to develop attention-related or externalising behavioural problems. ${ }^{42} 43$ Earlier studies showed that individuals with internalising problems appeared to report more negative cognitive disturbances or distortions than those with externalising problems. ${ }^{44}$ It has been reported that internalising and externalising problems were predictors of suicidal behaviours in youth, and internalising problems may have a greater effect size for suicidality. ${ }^{45}$ Thus, it is reasonable to infer that due to possible chronic stress caused by societal discrimination and internalised heterosexism, sexual minority individuals are at greater risk of emotional and behavioural problems, which were risk factors for suicidal behaviours, and girls with emotional and behavioural problems might have elevated risk of suicidality due to increased negative influence on their motivation and cognitive processes.

Therefore, detecting and managing these symptoms among SMAs could be a crucial strategy for preventing suicidality in different sexes. First, related government departments should coordinate with public health organisations to devise strategies for diminishing social discrimination against sexual minorities. Second, families and schools are recommended to identify adolescents with emotional and behavioural problems as early as possible, and provide SMAs who experience these problems with appropriate support (eg, inviting children's openly sexual minority friends to family activities; supporting student clubs and activities, including homosexuality alliances against defamation and multicultural clubs $)^{46} 47$ to help them achieve greater self-esteem and health status. Third, psychologists, psychiatrists and clinical workers should develop and apply more effective emotional interventions (eg, improving emotional regulation capacity) ${ }^{48}$ and behavioural interventions (eg, dialectical behaviour therapy) ${ }^{49}$ targeting SMAs who have emotional and behavioural problems to promote their adaptive functioning and social interrelationships.

Indeed, some limitations should be considered when interpreting the results. First, we are only able to report adolescents' sexual minority status using a binary definition of sex (heterosexuals and sexual minorities), and gender dysphoria/transgender status was not assessed in this study, although the sexual minority status definition here is commonly used. A more detailed classification of sexual minority status should be included in future research. Second, due to the cross-sectional design, our approach to analysis cannot make causal inferences, despite allowing statistical prediction. A longitudinal study of the above relationships would be an optimal approach. Despite these limitations, the study presents the first known results on the mediating role of emotional and behavioural problems and the modulating role of sex in the associations of sexual minority status with suicidal behaviours using a large sample size of Chinese adolescents.

\section{CONCLUSIONS}

Our study uncovered the mediating roles of emotional and behavioural problems in the relationship between sexual minority status and suicidal behaviours in Chinese youth. On the whole, emotional and behavioural problems significantly and partially mediated the association between sexual minority status and suicidal behaviours among Chinese adolescents. Moreover, sex assigned at birth moderated these mediating effects, with a more significant effect seen among sexual minority girls than boys. To help prevent suicidality in SMAs, policymakers, educators, families and the overall community should work collaboratively to establish a safe social environment that embraces diversity and rejects stigmatisation. Moreover, the risks of emotional and behavioural problems in SMAs should be identified at an early stage and reduced through preventive measures to avoid suicidal behaviours.

Acknowledgements The authors would like to thank all the participants, the participating schools and the Department of Education for their assistance and support. In addition, the authors gratefully acknowledge technical support from the Guangdong ADR Monitoring Center.

Contributors CL conceptualised and designed the study, reviewed and revised the manuscript, and approved the final manuscript as submitted. WL conceptualised and designed the study, coordinated and supervised data collection, carried out the initial analyses, drafted the initial manuscript, and approved the final manuscript as submitted. LG carried out the analyses and interpreted the data, reviewed and 
revised the manuscript, and approved the final manuscript as submitted. SZ, WW, $\mathrm{XC}$ and QL designed the data collection instruments, coordinated and supervised data collection, reviewed and revised the manuscript, and approved the final manuscript as submitted. All authors approved the final manuscript as submitted and agree to be accountable for all aspects of the work.

Funding This work was supported by the Natural Science Foundation of Guangdong Province (grant no. 2018A0303130331), the National Natural Science Foundation of China (grant no. 81761128030), and the Guangdong Food and Drug Administration (grant no. GZSW11175FT4055).

Competing interests None declared.

Patient consent for publication Not required.

Ethics approval The study was approved by the Sun Yat-Sen University School of Public Health Institutional Review Board (research ethics approval number: L2014076).

\section{Provenance and peer review Not commissioned; externally peer reviewed.}

Data availability statement Data are available upon reasonable request. The data used to support the findings of this study are available from the corresponding author upon request.

Supplemental material This content has been supplied by the author(s). It has not been vetted by BMJ Publishing Group Limited (BMJ) and may not have been peer-reviewed. Any opinions or recommendations discussed are solely those of the author(s) and are not endorsed by BMJ. BMJ disclaims all liability and responsibility arising from any reliance placed on the content. Where the content includes any translated material, BMJ does not warrant the accuracy and reliability of the translations (including but not limited to local regulations, clinical guidelines, terminology, drug names and drug dosages), and is not responsible for any error and/or omissions arising from translation and adaptation or otherwise.

Open access This is an open access article distributed in accordance with the Creative Commons Attribution Non Commercial (CC BY-NC 4.0) license, which permits others to distribute, remix, adapt, build upon this work non-commercially, and license their derivative works on different terms, provided the original work is properly cited, appropriate credit is given, any changes made indicated, and the use is non-commercial. See: http://creativecommons.org/licenses/by-nc/4.0/.

\section{ORCID iD}

Ciyong Lu http://orcid.org/0000-0002-3524-9784

\section{REFERENCES}

1 WHO. Suicide, 2021. Available: https://www.who.int/news-room/ fact-sheets/detail/suicide [Accessed September 9, 2021].

2 Prinstein MJ, Nock MK, Simon V, et al. Longitudinal trajectories and predictors of adolescent suicidal ideation and attempts following inpatient hospitalization. J Consult Clin Psychol 2008;76:92-103.

3 Xu RB, Wen B, Song Y, et al. [The change in mortality and major causes of death among Chinese adolescents from 1990 to 2016] Zhonghua Yu Fang Yi Xue Za Zhi 2018;52:802-8.

4 Meyer IH. Prejudice, social stress, and mental health in lesbian, gay, and bisexual populations: conceptual issues and research evidence. Psychol Bull 2003;129:674-97.

5 Montoro R, Thombs B, Igartua KJ. [The association of bullying with suicide ideation, plan, and attempt among adolescents with GLB or unsure sexual identity, heterosexual identity with same-sex attraction or behavior, or heterosexual identity without same-sex attraction or behavior]. Sante Ment Que 2015;40:55-75.

6 Marshal MP, Dietz LJ, Friedman MS, et al. Suicidality and depression disparities between sexual minority and heterosexual youth: a metaanalytic review. J Adolesc Health 2011;49:115-23.

7 di Giacomo E, Krausz M, Colmegna F, et al. Estimating the risk of attempted suicide among sexual minority youths: a systematic review and meta-analysis. JAMA Pediatr 2018;172:1145-52.

8 Boonchooduang $\mathrm{N}$, Louthrenoo $\mathrm{O}$, Likhitweerawong $\mathrm{N}$, et al. Emotional and behavioral problems among sexual minority youth in Thailand. Asian J Psychiatr 2019;45:83-7.

9 Martin-Storey A, Lemelin J-P, Temcheff C, et al. Exploring childhood temperament as a moderator of the association between adolescent sexual minority status and internalizing and externalizing behavior problems. J Adolesc 2020;84:190-9.

10 Kovess-Masfety V, Pilowsky DJ, Goelitz D, et al. Suicidal ideation and mental health disorders in young school children across Europe. J Affect Disord 2015;177:28-35.
11 Zhao M, Xiao D, Wang W, et al. Association among maltreatment, bullying and mental health, risk behavior and sexual attraction in Chinese students. Acad Pediatr 2021;21:849-57.

12 Zhang $\mathrm{H}$, Wong WCW, Ip $\mathrm{P}$, et al. Health status and risk behaviors of sexual minorities among Chinese adolescents: a school-based survey. J Homosex 2017;64:382-96.

13 Li P, Huang Y, Guo L, et al. Sexual attraction and the nonmedical use of opioids and sedative drugs among Chinese adolescents. Drug Alcohol Depend 2018;183:169-75.

14 Huang Y, Li P, Lai Z, et al. Association between sexual minority status and suicidal behavior among Chinese adolescents: a moderated mediation model. J Affect Disord 2018;239:85-92.

15 Campaign HR. Glossary of terms 2021, 2021. Available: http://www. hrc.org/resources/glossary-of-terms

16 Juster R-P, de Torre MB, Kerr P, et al. Sex differences and gender diversity in stress responses and allostatic load among workers and LGBT people. Curr Psychiatry Rep 2019;21:110.

17 Plöderl M, Tremblay P. Mental health of sexual minorities. A systematic review. Int Rev Psychiatry 2015;27:367-85.

$18 \mathrm{Li} \mathrm{L}$, Orleans M. Coming out discourses of Asian American lesbians. Sexuality and Culture 2001;5:57-78.

19 Zheng L, Hart TA, Noor SW, et al. Stressors based on sexual orientation and mental health among Lesbian, gay, and bisexual individuals in China: minority stress and perceived pressure to get married. Arch Sex Behav 2020;49:1769-82.

20 Guo L, Wang W, Wang T, et al. Association of emotional and behavioral problems with single and multiple suicide attempts among Chinese adolescents: modulated by academic performance. J Affect Disord 2019;258:25-32.

21 Russell ST, Joyner K. Adolescent sexual orientation and suicide risk: evidence from a national study. Am J Public Health 2001;91:1276-81.

22 Roberts R, Black G, Hart T. Same-sex-attracted adolescents in rural Australia: stressors, depression and suicidality, and barriers to seeking mental health support. Rural Remote Health 2018;18:4364.

23 Guo L, Xu Y, Deng J, et al. Association between nonmedical use of prescription drugs and suicidal behavior among adolescents. JAMA Pediatr 2016;170:971-8.

24 Goodman R, Meltzer H, Bailey V. The strengths and difficulties questionnaire: a pilot study on the validity of the self-report version. Eur Child Adolesc Psychiatry 1998;7:125-30.

25 Goodman R, Meltzer H, Bailey V. The strengths and difficulties questionnaire: a pilot study on the validity of the self-report version. Int Rev Psychiatry 2003;15:173-7.

$26 \mathrm{Du}$ Y, Kou J, Coghill D. The validity, reliability and normative scores of the parent, teacher and self report versions of the strengths and difficulties questionnaire in China. Child Adolesc Psychiatry Ment Health 2008;2:8.

27 Liang L, Yang J, Yao S. Measurement equivalence of the SDQ in Chinese adolescents: a horizontal and longitudinal perspective. $J$ Affect Disord 2019;257:439-44.

28 Huang Y, Li P, Guo L, et al. Sexual minority status and suicidal behaviour among Chinese adolescents: a nationally representative cross-sectional study. BMJ Open 2018;8:e020969.

29 Baiden P, Mengo C, Boateng GO, et al. Investigating the association between age at first alcohol use and suicidal ideation among high school students: evidence from the youth risk behavior surveillance system. J Affect Disord 2019;242:60-7.

30 Radloff LS. The CES-D scale: a self-report depression scale for research in the general population. Appl Psychol Meas 1977;1:385-401.

31 Zhang J, Z-Y W, Fang G. Development of the Chinese age norms of CES-D in urban area. Chinese Mental Health Journal 2010;24:139-43.

32 Wang M, Armour C, Wu Y, et al. Factor structure of the CES-D and measurement invariance across gender in mainland Chinese adolescents. J Clin Psychol 2013;69:966-79.

33 Yang X, Lau JTF, Lau MCM. Predictors of remission from probable depression among Hong Kong adolescents - A large-scale longitudinal study. J Affect Disord 2018;229:491-7.

34 Huang R, Ho SY, Wang MP, et al. Sociodemographic risk factors of alcohol drinking in Hong Kong adolescents. J Epidemiol Community Health 2016;70:374-9.

35 Muthén L, Muthén B. Mplus user's guide (eight edition)[Computer software manual]. Los Angeles, CA 2017.

36 Klein A, Moosbrugger H. Maximum likelihood estimation of latent interaction effects with the LMS method. Psychometrika 2000;65:457-74

37 Garver MS, Mentzer JT. Logistics research methods: employing structural equation modeling to test for construct validity. Journal of business logistics 1999;20:33. 
38 Preacher KJ, Hayes AF. Asymptotic and resampling strategies for assessing and comparing indirect effects in multiple mediator models. Behav Res Methods 2008;40:879-91.

39 Ferrari AJ, Norman RE, Freedman G, et al. The burden attributable to mental and substance use disorders as risk factors for suicide: findings from the global burden of disease study 2010. PLoS One 2014;9:e91936.

40 DeCou CR, Lynch SM. Sexual orientation, gender, and attempted suicide among adolescent psychiatric inpatients. Psychol Serv 2018:15:363-9.

41 King M, Semlyen J, Tai SS, et al. A systematic review of mental disorder, suicide, and deliberate self harm in lesbian, gay and bisexual people. BMC Psychiatry 2008;8:70.

42 Garnefski N, Kraaij V, van Etten M. Specificity of relations between adolescents' cognitive emotion regulation strategies and internalizing and Externalizing psychopathology. J Adolesc 2005;28:619-31.

43 Tambelli R, Laghi F, Odorisio F, et al. Attachment relationships and internalizing and Externalizing problems among Italian adolescents. Child Youth Serv Rev 2012;34:1465-71.
44 Epkins CC. Cognitive specificity in internalizing and externalizing problems in community and clinic-referred children. $J$ Clin Child Psychol 2000;29:199-208.

45 Soto-Sanz V, Castellví P, Piqueras JA, et al. Internalizing and externalizing symptoms and suicidal behaviour in young people: a systematic review and meta-analysis of longitudinal studies. Acta Psychiatr Scand 2019;140:5-19.

46 Ryan C, Russell ST, Huebner D, et al. Family acceptance in adolescence and the health of LGBT young adults. J Child Adolesc Psychiatr Nurs 2010;23:205-13.

47 Wilson MN, Asbridge M, Langille DB. School connectedness and protection from symptoms of depression in sexual minority adolescents attending school in Atlantic Canada. J Sch Health 2018;88:182-9.

48 Rogers $\mathrm{AH}$, Seager I, Haines $\mathrm{N}$, et al. The indirect effect of emotion regulation on minority stress and problematic substance use in Lesbian, gay, and bisexual individuals. Front Psychol 2017;8:8.

49 Miller AL. Dialectical behavior therapy: a new treatment approach for suicidal adolescents. Am J Psychother 1999;53:413-7. 